\title{
reviscafuences
}

ISSN: 1575-7072 | e-ISSN: 2172-7775

Páginas: 127-141

Recibido: 2019-07-19

Revisado: 2019-12-05

Aceptado: 2020-01-14

Preprint: 2020-04-20

Publicación Final: 2020-06-15 www.revistascientificas.us.es/index.php/fuentes/index

DOI: https://doi.org/10.12795/revistafuentes.2020.v22.i1.10

\section{Motivación y concepciones a las que alumnos de educación básica atribuyen su rendimiento académico en matemáticas}

\section{Motivation and conceptions that elementary education students attribute to their academic performance in mathematics}

\author{
(iD) Miryam Sofía Corredor-García \\ Secretaría de Educación de Boyacá (Colombia) \\ Josefina Bailey-Moreno \\ Tecnologico de Monterrey (México)
}

\section{Resumen}

Este estudio tuvo como objetivo conocer en estudiantes de educación básica secundaria de Colombia, cómo es su motivación en matemáticas, e identificar las concepciones a las que atribuyen el rendimiento académico. La metodología cualitativa permitió acercarse a las vivencias y experiencias de treinta y un estudiantes y de la maestra de matemáticas a través de la observación y entrevistas. Entre los principales resultados se encontró que predomina la motivación extrínseca promovida por los padres de familia y maestra, mientras que la motivación intrínseca se manifiesta en la autorregulación y en el rendimiento académico satisfactorio y avanzado de cuatro estudiantes. Se encuentra que el ambiente del salón de clases no es favorable para el aprendizaje, aunque existen buenas relaciones interpersonales entre alumnos-alumnos y alumnos-maestra. La responsabilidad, comprensión y gusto por las matemáticas son aspectos atribuibles al buen rendimiento, mientras que el desorden, la indisciplina y la falta de atención a las explicaciones de la maestra son las atribuibles al bajo rendimiento. Se concluye que la motivación no garantiza un buen rendimiento académico, ya que parece tener más impacto el clima del aula y las estrategias docentes, lo que implica cambiar las concepciones y condiciones de enseñanza para el aprendizaje activo y con ello establecer nuevas relaciones con el conocimiento matemático.

\section{Abstract}

The objective of this study was to know in elementary education students from Colombia, what their motivation in mathematics is, and to identify the conceptions to which they attribute academic performance. The qualitative methodology allowed to approach the experiences of thirty-one students and the teacher's mathematics through observation and interviews. Among the main results, it was found that the extrinsic motivation promoted by the parents and teacher predominates, while the intrinsic motivation is manifested in the self-regulation and in the satisfactory and advanced mathematics achievement of four students. It is found that the classroom environment is not favorable for learning, although there are good interpersonal relationships between student-students and teacher-students. Responsibility, understanding and liking for mathematics are aspects attributable to good achievement, while disorder, indiscipline and lack of attention to the teacher's explanations are attributable to poor achievement. It is concluded that the motivation does not guarantee a good academic performance, since it seems to have more impact classroom climate and teaching strategies, which implies changing the conceptions and teaching conditions for active learning and thus establishing new relationships with mathematical knowledge.

\section{Palabras clave / Keywords}

motivación, rendimiento académico en matemáticas, aprendizaje activo, ambiente de aprendizaje, estrategias de enseñanza. motivation, mathematics achievement, active learning, learning environment, teaching strategies. 


\section{Introducción}

Este estudio se propuso conocer cómo es la motivación de los estudiantes en el área de matemáticas e identificar las concepciones a las que atribuyen su rendimiento académico. Se llevó a cabo en una institución educativa pública, ubicada en el municipio de Orocué, Casanare, Colombia, con una población de estudiantes de estrato socioeconómico bajo. De acuerdo con los resultados de la prueba SABER presentada por todos los grupos de estudiantes del grado octavo de básica secundaria, el rendimiento académico en la asignatura de matemáticas es preocupante, ya que de 78 estudiantes evaluados el $35 \%$ obtuvo un nivel de rendimiento insuficiente, el $41 \%$ en mínimo, $18 \%$ en satisfactorio y el $6 \%$ en un nivel avanzado. Así también, los estudiantes del grado octavo-grupo B, que participaron en este estudio, presentaron la prueba Supérate con la prueba SABER en septiembre del 2017 , de los cuales el $44 \%$ obtuvo un nivel de rendimiento insuficiente, $56 \%$ mínimo y ninguno en los niveles satisfactorio y avanzado (Instituto Colombiano para el Fomento de la Educación Superior, 2017).

Es conocido que las pruebas institucionales proporcionan información cuantitativa de los resultados de aprendizaje sin considerar las experiencias individuales y las condiciones del contexto social y escolar en las que ocurre la enseñanza. Este estudio adquiere relevancia dado que indaga cualitativamente diversos aspectos que participan en el proceso de enseñanza aprendizaje y rendimiento académico, lo cual contribuye a tener una visión comprensiva de dichos resultados. De entre los aspectos que intervienen en el proceso educativo se encuentra la manera en la que el docente atiende los aspectos académicos y disciplinarios (Del Valle-López, 2009), la forma en que los padres de familia se involucran en el desarrollo de los procesos de enseñanza (Elias, 2003), las relaciones interpersonales entre alumnos y docentes (González-Fernández, 2007). En este proceso también intervienen las estrategias pedagógicas, la empatía, las actitudes de respeto, atención, cuidado, paciencia y la motivación como eje central del ambiente de aprendizaje que el docente proporcione (Alonso-Tapia, 2011; Deci y Ryan, 2015 y Del Valle-López, 2009).

Debido a los múltiples aspectos que interactúan e influyen en los procesos motivacionales y el rendimiento académico, en este estudio se abordan tres modelos motivacionales 1) la teoría de la autodeterminación (TAD), la cual distingue entre motivación intrínseca y extrínseca en función del grado de internalización y autonomía de las motivaciones, y a su ausencia como amotivación, 2) la motivación social para el abordaje del papel que juegan los docentes, compañeros de clase y padres de familia como actores motivacionales y 3) la teoría de la atribución causal para la comprensión de las causas por las cuales los alumnos consideran obtener determinado rendimiento académico.

Diversos investigadores coinciden en definir a la motivación como una fuerza o energía interna o externa que mueve a la acción, es decir que impulsa a los estudiantes a lograr un objetivo determinado (Arana- Martínez, García-Meilán, Gordillo-León, y Carro-Ramos (2010); Deci y Ryan, 2015; Maquilón-Sánchez y HernándezPina, 2011; Naranjo-Pereira, 2009). Se ha comprobado que cuando se valoran y buscan objetivos intrínsecos como el crecimiento personal, las personas tienden a satisfacer tres necesidades básicas, de acuerdo con la TAD son los nutrientes para facilitar la motivación intrínseca y el bienestar, estas necesidades son: competencia (sentirse capaz); autonomía (controlar las propias acciones) y tener relaciones significativas. Mientras que la búsqueda de objetivos extrínsecos como el reconocimiento social y las recompensas materiales tienden a inhibir dichas necesidades (Deci y Ryan, 2015). Las teorías motivacionales destacan la influencia del contexto social y escolar como facilitadores de diferentes tipos de motivación.

La motivación intrínseca de acuerdo con Arana et al. (2010) y González-Fernández (2007), es aquella fuerza que se encuentra en el interior de cada persona y que impulsa a fijar y obtener metas y proyectos para la obtención de sus sueños. En el ámbito educativo, la motivación intrínseca la experimentan aquellos estudiantes que tienen altas expectativas por aprender y son persistentes a la hora de llevar a cabo sus actividades académicas puesto que disfrutan realizándolas, viéndose esto reflejado en su rendimiento académico (Valenzuela y Nieto, 2008; Valle, Rodríguez, Núñez, Cabanach, González-Pienda y Rosario, 2010). Deci y Ryan (2015) señalan que, en este tipo de motivación, el interés y disfrute produce en los estudiantes aprendizajes más profundos y altos niveles de comprensión conceptual, ya que "están haciendo lo que encuentran interesante hacer, en el proceso están aprendiendo y creciendo" (p.487). Para estos mismos autores este tipo de motivación es el prototipo de la motivación autónoma porque hay un sentido de voluntad en los comportamientos.

De igual manera, los estudiantes que permanecen intrínsecamente motivados tienden a experimentar un aprendizaje autorregulado, entendido como el proceso mediante el cual llevan a cabo la formulación, planeación, observación, activación y evaluación de las metas, permitiendo así el control de sus propios pensamientos y comportamientos, el desarrollo de habilidades y estrategias para dar solución a diversos 
problemas y con ello la competencia de aprender a aprender (Salmerón-Pérez y Gutiérrez-Braojos, 2012; Schunk, 2008; Valle et al., 2010).

La motivación extrínseca de acuerdo con la TAD puede explicarse mediante el proceso de internalización de las motivaciones como un proceso continuo hacia la autonomía, en tanto los motivos externos sean regulados e integrados en el sentido de sí mismo, de acuerdo con este proceso se han documentado cuatro tipos: la regulación externa, introyectada, identificada y la integrada (Deci y Ryan, 2015).

La regulación externa se origina en el contexto en el cual se desarrolla el estudiante, consiste en ofrecer estímulos o recompensas, ya sea por parte del maestro o de los padres de familia, es el tipo de motivación más controlada y por lo tanto menos autónoma, ya que los estudiantes responden o se comportan ante los estímulos o eventos externos que no han sido internalizados, es decir no han sido asumidos como propios (Deci y Ryan, 2015: Maquilón-Sánchez y Hernández-Pina, 2011). En la regulación introyectada los motivos o aspiraciones externas se asimilan en tanto refuerzan al ego, a la autoestima o evitan la culpa, pero la motivación sigue siendo controlada dado que los comportamientos no se asumen como propios en la búsqueda de la autonomía (Deci y Ryan, 2015). Ambos tipos pueden generar un efecto negativo en el desempeño académico porque los alumnos tienden a memorizar los conocimientos, además pueden volverse dependientes y controlados por estos eventos y con ello frustrar la necesidad de autonomía personal (Deci \& Ryan, 2015: Maquilón-Sánchez y Hernández-Pina, 2011).

La regulación identificada implica la internalización de motivos externos porque las personas se identifican al reconocer la importancia de los comportamientos para sí mismos y por consiguiente los asumen como propios. Finalmente, la regulación integrada consiste en la integración de las identificaciones con otros aspectos de la personalidad que dan sentido de sí mismo, por lo tanto, los motivos se asumen como propios y las personas actúan con voluntad y elección. Ambos tipos de regulación, la identificada e integrada junto con la motivación intrínseca se consideran formas autónomas de motivación en contraste con su ausencia o amotivación, la cual se presenta cuando las personas no se sienten capaces de llevar a cabo algún comportamiento o cuando no valoran la importancia de dicho comportamiento (Deci y Ryan, 2015).

La motivación social está relacionada con el rol que cumplen los padres de familia, docentes y compañeros en cuanto al apoyo y relaciones interpersonales que se establecen fuera y dentro del aula de clase, este tipo de motivación puede favorecer o inhibir la satisfacción de las necesidades de autonomía, competencia y relaciones (Deci y Ryan, 2015) y producir que el estudiante experimente un alto o bajo rendimiento académico (Gonzáles, 2007).

Los padres de familia pueden influir considerablemente en la motivación del estudiante, de acuerdo con González-Fernández (2007), el apoyo que estos proporcionen en los procesos de aprendizaje es uno de los aspectos fundamentales para que los alumnos tengan un buen rendimiento escolar. Por el contrario, cuando se carece de este apoyo, se afecta la realización de las tareas, el interés y el entusiasmo, lo que conlleva a que el estudiante presente un bajo rendimiento que puede incluso conducir a la deserción escolar (Gonzáles, 2007). En el mismo sentido, la influencia motivacional por parte de los compañeros de clase está directamente relacionada con el tipo de relaciones interpersonales que se establecen entre ellos, así como los procesos de elección y socialización, los cuales intervienen en el grado de satisfacción o de estrés que se vive en la escuela, en el rendimiento académico, incluso la permanencia en el colegio (Gonzáles, 2007).

Teniendo en cuenta que el rol del maestro en la motivación de los estudiantes es fundamental para el óptimo proceso de enseñanza-aprendizaje. Diversos investigadores señalan que para que este se lleve a cabo, el docente necesita asumir las siguientes condiciones:

- Desempeñar la labor docente con humor, comprensión, entusiasmo, amor, alentando y estimulando a los estudiantes para que ellos puedan aprender la empatía, la comunicación, la creatividad y el pensamiento crítico (Allen, 2013).

- Mostrar siempre actitudes de respeto, escucha, cuidado y paciencia en el aula y fuera de esta, para que los niños y adolescentes se sientan atendidos. Esto hace que en ellos aumente la confianza y permite un mejor desarrollo de su personalidad (Alonso-Tapia, 2011; FernándezDomínguez, 2005).

- Ser una persona pensante y comprometida que trabaja de la mano con la evolución de la ciencia, la tecnología y los procesos sociales, ya que la práctica docente es una actividad que está en un proceso de mejora y cuya finalidad es la formación de personas con determinadas habilidades (Flores y González, 2014).

- Desarrollar innovación educativa, utilizar metodologías activas y trabajar colaborativamente con otros docentes (Abellán-Toledo y Herrada Valverde, 2016). 
De acuerdo con la TAD cuando los contextos sociales, ya sean hogares, escuelas y salones de clase satisfacen las tres necesidades básicas de los estudiantes, estos tienden a "desarrollar una orientación de autonomía relativamente fuerte" (p.490), ya que se identifican con la importancia de realizar el trabajo escolar y por consiguiente internalizan ese valor. Cuando el contexto favorece la satisfacción de las necesidades de competencia y relación, pero frustra la autonomía, los estudiantes tienden a desarrollar una motivación controlada (externa e introyectada), mientras que cuando todas las necesidades son frustradas se desarrolla la amotivación (Deci y Ryan, 2015).

El rendimiento académico es definido por diversos investigadores como el resultado del proceso de evaluación que realiza el docente y que determina si el estudiante ha logrado los objetivos previamente establecidos (Erazo-Santander, 2011; León, 2008; Maquilón-Sánchez y Hernández-Pina, 2011; Morales, Morales y Holguin, 2016).

Para Erazo-Santander (2011), el rendimiento académico es el sistema que mide si los logros propuestos han sido alcanzados satisfactoriamente por el estudiante a través de diferentes estrategias educativas que han sido evaluadas a lo largo del proceso educativo. Morales et al. (2016) lo definen como la medición de los resultados de la eficacia de los procesos educativos en donde maestros, alumnos y escuelas, entre otros, están directamente involucrados pues inciden directa e indirectamente en el éxito o el fracaso del rendimiento académico. Para Maquilón y Hernández (2011), es el conjunto de destrezas, habilidades, intereses e ideales que emplea el estudiante para aprender y sacar adelante determinados objetivos, los cuales han de ser evaluados por el docente en el transcurso del proceso de enseñanza-aprendizaje. León (2008) lo define como el resultado satisfactorio que experimenta un estudiante al final de un proceso de aprendizaje producto de su esfuerzo, el cual estuvo enfocado en la realización de actividades con el fin de alcanzar unos logros determinados.

Pese a que existen diversas definiciones acerca de rendimiento académico, todas apuntan de una $u$ otra manera al resultado obtenido por el estudiante al finalizar el proceso de enseñanza-aprendizaje, el cual ha sido orientado por el maestro con el fin de alcanzar los objetivos propuestos. En el rendimiento académico intervienen diferentes aspectos, Miñano y Castejón (2008) señalan que la opinión que tenga el estudiante sobre sí mismo es una de las características que está relacionada con la personalidad y tiene incidencia en el rendimiento académico de los alumnos. Erazo-Santander (2011) considera que las características de la familia de la cual proviene el estudiante y los lazos que está presente con la institución educativa, así como los hábitos de estudio en cuanto a tiempo, organización y el cumplimiento de normas que tenga el estudiante, son elementos que están asociados con el rendimiento académico. Para Morales et al. (2016) el ambiente escolar que proporcione el maestro, los contenidos a desarrollar, la metodología y los recursos didácticos que utilice para llevar a cabo las clases, así como la infraestructura con la que cuente la escuela y Lamas (2015) considera que la baja motivación, el poco interés, la baja autoestima y las malas relaciones que se establezcan entre alumno y maestro pueden generar en el estudiante un bajo rendimiento académico.

Aunado a los diversos aspectos que intervienen en el rendimiento académico, destaca la importancia de examinar las causas a las que los estudiantes atribuyen sus resultados de aprendizaje. Weiner (1986) afirma que las causas percibidas de éxito y fracaso, es decir las razones por las cuales se considera que el objetivo fue o no alcanzado tienen consecuencias en las expectativas, los afectos y parecen influir en la motivación. Estas causas comparten tres propiedades comunes: locus, estabilidad y controlabilidad, las cuales se traducen en tres dicotomías, esto es la atribución causal a factores internos-externos, estables-inestables y controlables o no controlables.

Weiner (1986) destaca que el éxito percibido debido a causas internas como tener habilidades o haberse esforzado aumentan la autoestima, mientras que la atribución de fracaso a causas internas como la falta de habilidades o desinterés la disminuye. Esto significa que los afectos son influenciados por la propiedad de locus (factores internos y externos). Mientras que la expectativa del éxito después de un resultado es influenciada por la estabilidad de la causa, por ejemplo, si el resultado es atribuido a una causa estable como sostener el esfuerzo (no cambia), entonces el resultado se podrá anticipar con mayor seguridad, pero si el fracaso es atribuido al poco esfuerzo realizado como causa inestable (puede cambiar) entonces la persona puede anticipar esforzarse más en el futuro para lograr el éxito. Estos factores pueden percibirse controlables como la falta de atención, o no controlables como la falta de preparación del maestro (Weiner, 1986).

La dinámica en las interacciones entre estas tres dicotomías puede producir diferentes interpretaciones del éxito o fracaso. Por ejemplo, Pozo (2006) señala que, si la atribución de fracaso se hace a factores externos y estables relativos a la forma de enseñar del maestro, la expectativa del éxito disminuirá, mientras que la atribución del fracaso a un factor inestable y controlable la expectativa de éxito aumentará. Para este mismo 
autor atribuir el éxito a factores internos, estables y controlables y los fracasos a factores externos, inestables y no controlables representa el estilo de los alumnos que tienden a tener éxito y estar motivados.

\section{Metodología}

El estudio se llevó a cabo con un enfoque cualitativo, su propósito fue comprender cómo los estudiantes y la docente interpretan sus experiencias, cómo construyen su propia realidad partiendo de sus vivencias (Merriam, 2015 y Taylor y Bogdan, 2006) y el significado que desde su perspectiva tiene la motivación en la clase de matemáticas y la manera en la que consideran interviene en el rendimiento académico.

Participaron 31 estudiantes ( 15 hombres y 16 mujeres) del grado octavo de básica secundaria con edades de entre 12 y 15 años y la docente de matemáticas. La docente es Licenciada en Matemáticas y Física, tiene 27 años de edad y posee dos años de experiencia laboral como maestra en la institución.

Las técnicas empleadas para recoger la información fueron entrevistas semiestructuradas, observación no participantes y cuestionario. El cuestionario se elaboró con preguntas abiertas y fue respondido por los estudiantes en forma autoadministrada. La utilización inicial de este instrumento compuesto por 24 preguntas, tuvo como propósito conocer los puntos de vista de los estudiantes acerca de los motivos por los cuales estudian, el ambiente académico en la clase de matemáticas, la forma de enseñar, acompañar y evaluar de la docente, así como las relaciones que establecen entre compañeros y docente, la participación de sus padres en el proceso de aprender y sus consideraciones acerca del rendimiento académico. Esta información permitió diseñar los guiones de entrevistas con el propósito de ahondar en los temas y también posibilitó llevar a cabo el proceso de enumeración de las regularidades LeComte y Goetz (1988).

Para las entrevistas semiestructuradas se elaboraron guiones con preguntas abiertas que permitieron flexibilidad y oportunidad de profundizar en los temas abordados (Bernal, 2010). Se entrevistó a la maestra de matemáticas con apoyo de un guión con 18 preguntas y a todos los estudiantes en la modalidad focus group utilizando un guión compuesto por 20 preguntas, los estudiantes se organizaron tres grupos, dos de 10 integrantes y uno de 11. La observación no participante se utilizó para comprender mejor la cultura del grupo estudiado (Valenzuela y Flores, 2012). Se observaron tres clases de matemáticas con duración de 55 minutos cada una., se utilizó una bitácora para registrar las actividades realizadas en el aula y las interacciones de los participantes.

Para el análisis de los datos se realizó la transcripción textual de la información obtenida y a continuación se procedió a realizar la codificación, este proceso se llevó a cabo a partir de los incidentes o temas que fueron surgiendo y se clasificaron, para posteriormente agruparlos en categorías (Merriam, 2015). De este modo, los resultados se organizaron en cuatro categorías: motivación intrínseca y autorregulación; motivación extrínseca: regulación externa e introyectada; motivación social y concepciones a las que los estudiantes atribuyen su rendimiento académico. Este proceso se esquematiza en la tabla 1.

\section{Tabla 1}

Categorías, incidentes, fuentes e instrumentos de recolección de datos

\begin{tabular}{|c|c|c|c|c|}
\hline \multirow{2}{*}{\begin{tabular}{|cr} 
Categorías e incidentes & $\begin{array}{r}\text { Instrumentos } \\
\text { Fuentes }\end{array}$ \\
\end{tabular}} & Cuestionario & Entrevista & Observación & Entrevista \\
\hline & \multicolumn{2}{|c|}{ Alumnos } & Alumnos-docente & Docente \\
\hline $\begin{array}{l}\text { Motivación intrínseca y autorregulación } \\
\text { Incidentes: Interés por aprender. } \\
\text { Planeación en la realización de actividades. } \\
\text { Deseo de entender, saber más. } \\
\text { Importancia de las matemáticas en la vida } \\
\text { académica y cotidiana. } \\
\text { Control del aprendizaje. }\end{array}$ & $\mathrm{x}$ & $\mathrm{x}$ & $\mathrm{x}$ & $\mathrm{x}$ \\
\hline $\begin{array}{l}\text { Motivación extrínseca: regulación } \\
\text { externa e introxectada } \\
\text { Incidentes: Estudiar para recibir premios } \mathrm{y} / \mathrm{o} \\
\text { evitar castigos. } \\
\text { Evitar reprobar la materia. } \\
\text { Cumplir con expectativas de los padres de } \\
\text { familia. }\end{array}$ & $\mathrm{x}$ & $\mathrm{x}$ & & $\mathrm{x}$ \\
\hline $\begin{array}{l}\text { Motivación social } \\
\text { Incidenter. Relaciones interpersonales } \\
\text { alumnos-alumnos, alumnos-docente. } \\
\text { Ambiente de aprendizaje. } \\
\text { Apoyo de los padres de familia. }\end{array}$ & $\mathbf{x}$ & $\mathrm{x}$ & $\mathbf{x}$ & $\mathbf{x}$ \\
\hline $\begin{array}{l}\text { Concepciones a las que se atribuye el } \\
\text { rendimiento académico } \\
\text { Incidentes: Nivel de rendimiento. } \\
\text { Percepción de las causas del rendimiento. } \\
\text { Retroalimentación y evaluación docente. }\end{array}$ & $\mathrm{x}$ & $\mathbf{x}$ & & $\mathrm{x}$ \\
\hline
\end{tabular}


En la presentación de los resultados se describe el significado de cada categoría y se incluyen comentarios textuales (datos) de los participantes que dan apoyo a la validez de los resultados (Taylor y Bogdan, 2006).

\section{Resultados}

\subsection{Motivación intrínseca y autorregulación}

En esta categoría se describen los resultados relacionados con los motivos internos que impulsan a los estudiantes a alcanzar sus metas en la clase de matemáticas, las actividades que los estudiantes realizan para cumplir sus objetivos. En la tabla 2 se sintetizan los principales incidentes encontrados en esta categoría.

\section{Tabla 2}

Incidentes encontrados en la categoría Motivación intrínseca y autorregulación

\begin{tabular}{|c|l|}
\hline Categoría & \multicolumn{1}{|c|}{ Incidentes } \\
\hline Motivación intrínseca y & Alumnos \\
autorregulación & Participar activamente en las clases \\
& Matemáticas es una asignatura importante \\
& Aprender \\
& Hacer los talleres \\
& Entregar bien los trabajos \\
& Nueve estudiantes gustan de las matemáticas y realizan sus \\
& actividades académicas porque entienden y aprenden más \\
& Nueve estudiantes controlan su propio aprendizaje \\
& 22 estudiantes no controlan su propio aprendizaje \\
& Maestra \\
& Explica, corrige errores, ofrece soluciones, no promueve estrategias \\
& de autorregulación \\
\hline
\end{tabular}

Los estudiantes cuya motivación intrínseca es elevada tienen interés por aprender y disfrutan cuando realizan determinadas tareas. En este caso nueve estudiantes manifestaron que estudian y llevan a cabo sus tareas porque aprenden más, porque entienden mejor, porque les gustan las matemáticas y la consideran una asignatura importante. Al respecto uno de los estudiantes manifestó: "Me gusta hacer mis tareas de matemáticas porque así me desarrollo más como estudiante". Dos estudiantes comentaron respectivamente:

Me motiva sacar adelante mis actividades en matemáticas porque esta asignatura es muy importante para mi estudio. Cumplir mis sueños, aprender cosas nuevas, ser alguien en la vida, entender la temática.

Dentro de las actividades que los estudiantes realizan para cumplir sus objetivos están solucionar los problemas que la profesora deja para la casa o para desarrollar en el salón, consultar más acerca de lo que la maestra explica, estudiar para las evaluaciones y prestar atención en clase. En una de las entrevistas grupales los estudiantes manifestaron "cumplir con las tareas, prestando atención en clase y opinando acerca del tema, participando activamente, haciendo los ejercicios que la profesora plantea en el tablero para trabajar en clase".

Durante las clases la función que asumió la maestra fue explicar, orientar, corregir los posibles errores, hacer los llamados de atención necesarios para mantener la disciplina y el orden, pero no se observó que fomente la autorregulación en el aprendizaje de los estudiantes. La siguiente nota de observación muestra la forma en que la docente resuelve las inquietudes en la clase:

La docente en el momento en el cual algún estudiante plantea tener una inquietud, se desplaza hasta el puesto del mismo y de forma personalizada resuelve la duda que manifiesta tener. Él 
estudiante muestra sentirse conforme con la explicación y continúa desarrollando el ejercicio. (Nota de observación, 27 de febrero del 2018).

Respecto al proceso de autorregulación cuatro estudiantes manifestaron que a veces realizan las tareas en casa hasta donde las entienden, porque tienen otras cosas que hacer en la casa. Los 18 estudiantes restantes comentaron no realizar sus tareas porque no entienden, porque prefieren hacerlas en el salón cuando la profesora explica en el tablero, porque no les queda tiempo, porque tienen otras tareas que hacer, porque no les agrada las matemáticas, un estudiante comentó: "Realizo las tareas cuando entiendo, si no entiendo las dejo a medio terminar y en el salón las termino".

En este sentido, los datos sugieren que 22 estudiantes no controlan su propio aprendizaje, al no asumir por sí mismos esta responsabilidad.

En conclusión, nueve de los 31 estudiantes conceptualizan su motivación como intrínseca porque les gusta aprender matemáticas y esto los conduce a emplear el tiempo que sea necesario para hacer sus labores escolares de la mejor manera posible, lo que se ve directamente reflejado en la autorregulación o control por su propio aprendizaje.

\subsection{Motivación extrínseca: regulación externa e introyectada}

En esta categoría se describe el tipo de apoyo y las recompensas que prometen tanto los padres de familia como la maestra para ayudar a los estudiantes a cumplir con sus responsabilidades académicas. En la tabla 3 se describen los principales incidentes.

\section{Tabla 3}

Incidentes encontrados en la categoría Motivación extrínseca: regulación externa e introyectada

\begin{tabular}{|l|l|}
\hline \multicolumn{1}{|c|}{ Categoría } & \multicolumn{1}{|c|}{ Incidentes } \\
\hline Regulación externa & Alumnos \\
& 18 estudiantes reciben recompensas: celulares, computador, tablet, \\
bicicleta, patines, permisos para hacer deporte si obtienen \\
calificaciones aprobatorias. \\
22 estudiantes trabajan en la asignatura de matemáticas por evitar \\
malas calificaciones y reprobar. \\
13 estudiantes consideran que reciben premios porque se lo \\
merecen. \\
Maestra \\
Otorga puntos positivos o excelentes notas para motivar a los \\
estudiantes. \\
\\
Regulación introvectada \\
Alumnos \\
22 estudiantes estudian para aprobar la materia, obtener \\
reconocimiento y ser orgullo para sus padres. \\
22 estudiantes se comportan bien en clases para evitar les den quejas \\
a sus padres. \\
\end{tabular}

La motivación extrínseca se genera en el exterior del estudiante y esto lo conduce a realizar una tarea determinada. En relación a la regulación externa que es el tipo de motivación más controlada por eventos externos, 18 estudiantes manifestaron que sus padres les prometen y otorgan algún tipo de recompensa o premio con la condición de que lleven a cabo sus responsabilidades académicas, se porten bien y obtengan buenas calificaciones. Dentro de estas recompensas están: celulares, computador, tablet, bicicleta, patines, otorgar permiso para entrenar algún deporte. Al respecto un estudiante manifestó: "Pues mi mamá me dice que me porte bien y que saque buenas notas para que ella me dé el celular que quiero". Por su parte la maestra manifestó: 
En ocasiones otorgo puntos positivos o excelentes notas a los estudiantes que hagan las actividades porque es una forma de motivarlos a que estudien, así sea por la nota.

Trece estudiantes señalaron que sus padres les dan premios o estímulos porque se lo merecen ya que realizan bien las actividades escolares, un estudiante dijo: "Me dan algo porque me lo merezco, porque hago las cosas bien". Otro de los recursos que, de acuerdo con los estudiantes, utilizan los padres para motivarlos es que estudien para no tener que recursar la materia "Ellos nos dicen que nunca dejemos de estudiar porque si no, nos quedamos a recuperar y dañamos nuestras vacaciones".

Veintidós estudiantes comentaron que se sienten motivados para lograr sus objetivos en la asignatura de matemáticas por ser el orgullo para sus padres, comportarse bien para evitar que les den quejas, ser personas de bien y no tener malas calificaciones para no perder esta materia ni el año escolar. Al respecto un estudiante comentó: "Me motiva mi mamá, mi papá y sacar buena nota para seguir adelante". Otro estudiante señaló: "Mis papás dicen que no deje de estudiar para que sea una persona de bien". Los estudiantes en la entrevista grupal dijeron que dentro de las actividades que realizan en la clase de matemáticas están: "Portarnos bien en la clase de matemáticas para que no nos hagan acta de compromiso y para que no llamen a los padres de familia y les den quejas". Estos hallazgos sugieren que los motivos de los estudiantes siguen siendo regulados por el exterior, pero que de algún modo los han introyectado para evitar la culpa o como una forma de proteger al yo.

Este tipo de conductas indican que los estudiantes se conducen ante el aprendizaje para evitar algún castigo o lograr un beneficio que no está relacionado directamente con el aprendizaje de las matemáticas. El hecho de que los estudiantes reciban alguna recompensa por parte de sus padres de familia y de la maestra hace que esto tenga una incidencia poco favorable para su autonomía y crecimiento académico.

\subsection{Motivación social}

En esta categoría se presentan los resultados acerca de las relaciones interpersonales que establecen entre alumnos-maestra y alumnos-alumnos, los aspectos que a los estudiantes les agradan y desagradan cuando están en compañía de sus compañeros en la clase de matemáticas, las razones por las cuales estas situaciones les generan estrés o algún grado de insatisfacción y el apoyo que reciben de sus padres en las actividades académicas. En la tabla 4 se esquematizan los principales incidentes encontrados en la esta categoría.

\section{Tabla 4}

Incidentes encontrados en la categoría Motivación social

\begin{tabular}{|l|l|}
\hline \multicolumn{1}{|c|}{ Categoría } & \multicolumn{1}{c|}{ Incidentes } \\
\hline Motivación social & Alumnos \\
& Les agrada la buera convivencia, el buen trato, cuando existe \\
& respeto, orden y disciplina. \\
& Les desagrada la indisciplina, el irrespeto, el desorden, las groserías, \\
& las peleas y la irresponsabilidad. \\
& Cuando hay indisciplina en el salón experimentan estrés o algún \\
& grado de insatisfacción. \\
& Las relaciones interpersonales entre los compañeros son amigables \\
& Los estudiantes y la maestra mantienen buenas relaciones \\
& interpersonales. \\
& 23 estudiantes reciben apoyo académico de sus padres \\
& 4 estudiantes reciben apoyo académico esporádico de sus padres \\
& 4 estudiantes carecen de apoyo académicos de sus padres. \\
& Maestra \\
& Ambiente de aprendizaje con fallas en el control de la disciplina \\
\hline
\end{tabular}


La motivación social está relacionada con el rol que cumplen los padres, profesores y compañeros en la motivación de un alumno. Al respecto los estudiantes manifestaron que dentro de los aspectos que les agradan del ambiente en el salón durante la clase de matemáticas están la buena convivencia y el buen trato, trabajar juntos para sacar conclusiones y respuestas entre todos, les agrada que la maestra revise la tarea de manera personalizada y cuando en el salón de clases existe respeto, orden y disciplina. Algunos estudiantes comentaron: "Me agrada cuando estamos en la clase de matemáticas y ellos son muy disciplinados", "me agradan cuando estoy con mis compañeros en clase y opinamos para realizar el trabajo mejor", "cuando son amables y me respetan".

A 28 estudiantes les desagradan los siguientes aspectos durante las clases de matemáticas: la indisciplina, el irrespeto, el desorden, las groserías, que hablen mucho los compañeros y que no respeten la palabra, que se levanten del puesto sin autorización de la maestra, las peleas verbales entre los compañeros, la irresponsabilidad cuando no cumplen con sus obligaciones académicas (tareas). Algunos comentarios de los estudiantes al respecto: "El aspecto que me desagrada es la indisciplina porque si uno de mis compañeros comienza a molestar alguien más le sigue la corriente", "pues unas veces que dicen groserías, pelean, uno está trabajando, el salón está en silencio y no falta el bulloso", "lo que más me desagrada es que uno este concentrado y los demás estén haciendo desorden". La siguiente nota de observación ejemplifica el ambiente de clase.

Los estudiantes participan activamente en la clase, hacer preguntas y dan su punto de vista respecto al tema, sin embargo, en cuanto al clima de aula se observó poco control de la disciplina por parte de la maestra, ya que el alumno puede hablar con sus compañeros, levantarse de su silla y deambular por el aula mientras la maestra explica la temática (Nota de observación, 28 de febrero del 2018).

La maestra describe el comportamiento de los estudiantes de la siguiente manera: "Es un poco desesperante ya que hablan mucho, no escuchan lo que dicen, todos quieren opinar a la vez y muy poco se concentran en las clases".

Los estudiantes hablan entre sí de manera constante, a la maestra se le dificulta llevar a cabo las explicaciones, varios alumnos responden las preguntas que plantea la maestra al mismo tiempo, sin un orden establecido (Nota de observación, 27 de febrero del 2018).

La maestra de matemáticas también es la directora de grupo por lo que en sus clases se tratan temas relacionados con diferentes asuntos o problemáticas que al interior del mismo se generan. Estas acciones hacen que la maestra concentre parte del tiempo de clase en resolver aspectos disciplinarios y situaciones que no tienen que ver con el desarrollo de la clase. Estos eventos se presentaron en las tres observaciones, el tiempo promedio de clase efectiva fue de 25 minutos de 60 que dura la hora de clase.

Estas situaciones que se viven en el ambiente de clase generan en 27 estudiantes estrés o algún grado de insatisfacción, de acuerdo con lo manifestado por los alumnos llegan a sus casas cansados y con dolor de cabeza, entre los comentarios más representativos están "porque cuando hacen ruido no dejan concentrar a los que queremos trabajar juiciosamente, porque mi salón parece un salón de locos, porque a mis compañeros no se los aguanta nadie, porque mis compañeros no son serios, hacen demasiado ruido, y eso me da mal genio, porque definitivamente no hay confianza, porque mis compañeros son groseros cansones y fastidiosos y porque en ocasiones hace mucho calor". Al respecto algunos estudiantes comentaron: "Si porque en mi salón no sabemos lo que es el respeto y ofendemos, criticamos y todo eso me causa estrés", "estrés porque a veces hacen mucho ruido y no dejan concentrar a los que trabajan juiciosamente".

Por otro lado, 15 estudiantes manifestaron que las relaciones interpersonales que mantienen con sus compañeros de clase son buenas porque algunos son amigables, respetuosos, amables, porque se tratan bien y aprenden unos de otros, pero 16 estudiantes por el contrario señalaron que a veces no mantienen buenas relaciones interpersonales porque pelean entre ellos mismos, se expresan mal diciendo groserías y esto causa heridas en los sentimientos, pero después lo olvidan y vuelven a interactuar. Algunos estudiantes manifestaron: "Bien porque nos tratamos bien, a veces peleamos, pero después volvemos a estar como si nada", "Algunas veces discutimos, pero cuando ya ha pasado todo volvemos hablar".

El apoyo que 23 estudiantes reciben por parte de los padres de familia consiste en explicarles los temas que no entienden, darles dinero para que realicen consultas en internet, apoyarlos emocionalmente brindándoles amor, cariño y escucha, proporcionarles los útiles escolares que necesitan, brindándoles opiniones para que 
los trabajos les queden mejor, un estudiante comentó "mis padres sí me apoyan, dándome dinero para consultar en internet".

A cuatro estudiantes sus padres les brindan apoyo de manera esporádica puesto que mantienen mayormente su atención en su trabajo, y para cuatro estudiantes no existe el apoyo y hacen las tareas por sí mismos, al respecto algunos estudiantes comentaron: "Mi padre a veces sólo me explica y no tanto porque se la pasa enterado del trabajo y no de mi estudio. Ningún apoyo, por lo general hago solo las tareas". La maestra manifestó no conocer algún tipo de acompañamiento que los padres de familia lleven a cabo con los estudiantes.

Estos resultados informan que las interacciones en el salón de clases y el apoyo que los padres ofrecen a los estudiantes no facilitan el acceso al conocimiento. Durante las clases, la indisciplina genera baja atención y concentración a las instrucciones y explicaciones de la maestra, de las percepciones de los estudiantes y observaciones realizadas se encontró dificultad para que el aula sea un espacio propicio para el dialogo y conexión con el aprendizaje.

\subsection{Concepciones a las que los estudiantes atribuyen su rendimiento académico}

En esta categoría se presenta el rendimiento académico obtenido por los estudiantes a partir de la evaluación de la maestra, así como la valoración de los estudiantes de su propio rendimiento y sus concepciones respecto al mismo. En la tabla 5 se describen los principales incidentes encontrados en esta categoría.

\section{Tabla 5}

Incidentes encontrados en la categoría Concepciones a las que los estudiantes atribuyen su rendimiento académico

\begin{tabular}{|c|l|}
\hline Categoría & \multicolumn{1}{c|}{ Incidentes } \\
\hline $\begin{array}{c}\text { Concepciones } \\
\text { atribuibles al } \\
\text { rendimiento } \\
\text { académico }\end{array}$ & $\begin{array}{l}\text { Alumnos } \\
\text { El bajo nivel de rendimiento académico con calificaciones mínimas e } \\
\text { insuficientes en general son producto de la falta de comprensión a las } \\
\text { explicaciones de la maestra, falta de atención e indisciplina en el salón de } \\
\text { clases. }\end{array}$ \\
& $\begin{array}{l}\text { El rendimiento avanzado y satisfactorio son producto de la disciplina, } \\
\text { responsabilidad, buen comportamiento, compromiso consigo mismo. } \\
\text { Maestra } \\
\text { Realiza evaluaciones por medio de ejercicios para aplicar el } \\
\text { conocimiento } \\
\text { Señala el error y explica al grupo la solución correcta }\end{array}$ \\
\hline
\end{tabular}

La maestra manifestó que la estrategia que emplea para evaluar el rendimiento académico de los estudiantes es por medio de exámenes con ejercicios para que apliquen lo aprendido. De igual manera comentó que da a conocer los resultados de las evaluaciones de manera clara y oportuna llevando a cabo el siguiente procedimiento: "Le entrego a cada uno su evaluación, leo punto por punto, los desarrollo en el tablero y les explico qué debieron hacer para que la evaluación hubiera quedado bien resuelta".

La escala de valoración que se emplea en la institución educativa para medir el rendimiento académico es la siguiente: avanzado: 4.5 a 5.0, satisfactorio: 3.7 a 4.4, mínimo: 3.0 a 3.6 e insuficiente: 1.0 a 2.9. En la tabla 6 se presenta un comparativo entre el nivel de rendimiento académico obtenido por los estudiantes resultante de la evaluación de la maestra y el considerado por los alumnos sobre su propio rendimiento. 


\section{Tabla 6}

Nivel de rendimiento académico, comparativa entre la valoración de la maestra y autovaloración de alumnos.

\begin{tabular}{|l|c|c|}
\hline \multicolumn{1}{|c|}{ Cantidad alumnos } & Valoración maestra & Autovaloración alumnos \\
\hline Escala de valoración & & \\
\hline Avanzado & 2 & 3 \\
Satisfactorio & 2 & 15 \\
Mínimo & 25 & 12 \\
Insuficiente & 2 & 1 \\
\hline
\end{tabular}

Con base en la información obtenida del consolidado de calificaciones del primer periodo académico, se encontró que dos estudiantes están en el nivel avanzado, dos en el nivel satisfactorio, 25 estudiantes se encuentran en un nivel de desempeño mínimo y dos en insuficiente. La maestra señaló que "a nivel general los estudiantes se encuentran en un nivel de desempeño mínimo, pero hay chicos que están en satisfactorio porque trabajan como es debido".

Respecto a la concepción que tienen los estudiantes de su rendimiento y los aspectos a los que les atribuyen dicho rendimiento se encontró lo siguiente: 15 estudiantes consideraron estar en el nivel satisfactorio, atribuyen estar en ese nivel porque se portan bien en las clases y son responsables. Uno de los estudiantes manifestó: "Satisfactorio, porque soy un niño trabajador en el área y responsable con mis actividades". Tres estudiantes señalaron estar en el nivel avanzado porque entienden lo que la maestra explica y les gustan las matemáticas. Al respecto uno de los estudiantes manifestó: "Avanzado porque le echo ganas a las matemáticas, desarrollo los trabajos, soy muy juiciosa y no hago tanto desorden".

En el mismo sentido, un estudiante dijo estar en nivel insuficiente: "Porque casi no le entiendo a la profesora de matemáticas", 12 estudiantes atribuyen estar en un nivel mínimo porque a pesar de que en ocasiones desarrollan las actividades, consideran que deben aprender más para mejorar, pues poco le entienden a la maestra debido al desorden, la indisciplina y la escasa atención durante las clases. Por tales razones no llevan a cabo las tareas, trabajos y actividades académicas. Uno de los estudiantes que se encuentra en este nivel de desempeño dijo: "Mínimo, porque en algunas ocasiones no entiendo".

Estos hallazgos sugieren que los alumnos que perciben su rendimiento avanzado y satisfactorio atribuyen el éxito a su propio esfuerzo y dedicación, mientras que los estudiantes que perciben su rendimiento insuficiente y mínimo atribuyen las causas a factores externos como la falta de atención debido al desorden en el salón de clases y no entender las explicaciones de la maestra.

\section{Discusión}

Este estudio se propuso conocer cómo es la motivación de un grupo de estudiantes de básica secundaria en la clase de matemáticas e identificar a qué causas atribuyen su rendimiento académico, el interés se centró en abordarlo desde la visión cualitativa, escuchando las voces de los participantes, con la intención de entender desde su perspectiva cómo viven e interpretan los eventos en la clase. Si bien es cierto que la motivación y el rendimiento académico son dos procesos que están estrechamente relacionados, puesto que los estudiantes que están motivados tienden a tener comportamientos de mayor calidad y por consiguiente mayor rendimiento (Caso-Niebla y Hernández- Guzmán, 2007; Deci y Ryan, 2015). También es cierto que alumnos y docente piensan y actúan desde su bagaje cultural y desde la organización social e institucional en la cual están inmersos. Es entonces que a partir de la dinámica de los significados y desde la lógica entre autonomía y control, como se pretendió en este estudio aproximarse a la comprensión de los eventos que dan sentido a la motivación y rendimiento académico.

Cabe señalar que el grupo estudiado estuvo conformado por 31 alumnos de entre 12 a 15 años, inscritos en una escuela pública de nivel socioeconómico bajo, las clases de matemáticas se imparten cuatros veces por semana con duración de 60 minutos cada una. La maestra con Licenciatura en Matemáticas y Física también es la tutora o directora del grupo por lo que frecuentemente destina tiempo de su clase para tratar asuntos no relacionados con la asignatura. 
En cuanto a la motivación intrínseca se encontró que nueve alumnos en sus declaraciones señalaron que estudian para aprender, son dedicados, constantes y gustan de las matemáticas al considerar esta asignatura como una de las más importantes para su desarrollo académico y en la vida cotidiana. Esto coincide con Valenzuela y Nieto (2008) al afirmar que los estudiantes que poseen motivación intrínseca son perseverantes al otorgar a sus deberes escolares un alto grado de importancia. Así también estos comportamientos informan que en el estudiante se ha desarrollado un proceso de autonomía o autorregulación de su aprendizaje (Deci y Ryan, 2015; Valle et al., 2010). Cabe destacar que a partir de las perspectivas de los estudiantes no es posible establecer en todos los casos una relación entre motivación intrínseca y buen rendimiento académico, ya que cinco de estos nueve estudiantes obtuvieron desde la evaluación docente un rendimiento insuficiente. Los resultados sugieren que el rol de la maestra en este contexto no es el más adecuado para fomentar la autonomía en los estudiantes, ya que la cantidad de alumnos en el grupo, el tiempo destinado a la clase y el tener que cumplir con los contenidos del programa de estudios, hace que asuma el control de la clase explicando, aclarando dudas, resolviendo ejercicios y problemas aún y cuando los alumnos los realicen equivocadamente, estas acciones conllevan a que los estudiantes no tengan la oportunidad de reflexionar y autoevaluar sus procesos de aprender, lo cual pudiera facilitar la comprensión y sensación de control de sí mismos, requisito indispensable para sostener el esfuerzo constante en el aprendizaje (Alonso Tapia, 2011; Deci y Ryan, 2015; Shunck, 2008 y Zimmerman, 2010).

Respecto a la motivación del tipo regulación externa que en términos de Deci y Ryan (2015) que es la que ejerce más control sobre los comportamientos, se encontró que la manera en la que la maestra motiva a los estudiantes es otorgándoles puntos extras por la realización de las actividades, 18 estudiantes reciben estímulos o recompensas materiales como celulares, tablets y bicicletas entre otros, por parte de sus padres para que realicen las actividades académicas, esto hace suponer que ante las condiciones socioeconómicas de los estudiantes estos premios resulten ser buenos incentivos. En cuanto a la evitación de eventos percibidos como negativos, 22 estudiantes trabajan en la asignatura con el fin de evadir malas calificaciones y reprobar la asignatura. En relación a los comportamientos que los alumnos pudiesen haber asimilado para obtener reconocimiento social, evitar la culpa y protegerse a sí mismos o regulación introyectada (Deci y Ryan, 2015), se encontró que 22 alumnos estudian para que sus padres se sientan orgullosos, evitar recursar la materia en verano y con ello no perder las vacaciones, así como portarse bien en clases para que la maestra no elabore reportes por mal comportamiento. Esto sugiere que en la organización áulica, escolar y familiar prevalece la idea de controlar las acciones de los estudiantes, ya que no se observa la promoción del interés académico y procesos de autorregulación. Por el contrario, se fomenta en el estudiante la dependencia a los premios y evitación de castigos, lo que conduce a inhibir en los alumnos la comprensión de la importancia del estudio para el crecimiento personal (Deci y Ryan, 2015; Shunck, 2008).

En referencia a la motivación social o tipo de interacciones que se establecen en el aula y la influencia de los padres de familia en lo académico, se encontró que los padres actúan como proveedores emocionales y de materiales necesarios para el desarrollo de las tareas, mientras que las interacciones en el aula están enmarcadas por los episodios de indisciplina y desorden, la docente emplea 26 minutos en promedio de 50 solucionando inconvenientes de índole disciplinario, lo que afecta al desarrollo de la clase., esto coincide con lo descrito por León (2008) en cuanto a que la indisciplina y el no prestar atención a explicaciones de la maestra, dificulta que el estudiante logre llevar a cabo las actividades para alcanzar un resultado satisfactorio. Sin embargo, y a pesar del clima áulico desordenado, las relaciones interpersonales entre alumnos-alumnos y alumnos-maestra son consideradas positivas o amigables, estos hallazgos contradicen lo hallado por González-Fernández (2007) quien señaló que el rol que juegan las relaciones interpersonales que se establezcan entre estudiantes y docentes ayudan a mantener un buen ambiente de aprendizaje en el aula. Deci y Ryan (2015) señalan que cuando la escuela y familia no favorece en los estudiantes la autonomía, pero sí que se perciban a sí mismos capaces, al mismo tiempo que prevalecen relaciones interpersonales positivas, son contextos que promueven la motivación extrínseca. De los resultados de la motivación extrínseca y social se pueden inferir que para los estudiantes ser competente en este contexto tiene sentido en tanto los comportamientos respondan a las exigencias externas y a la dependencia de ser controlado, esto pudiera explicar el desarrollo de la motivación extrínseca en la mayoría de los estudiantes de este grupo En relación a las concepciones a las que los estudiantes atribuyen su buen rendimiento académico, se encontró la dedicación del tiempo necesario para cumplir sus actividades de manera responsable, así como el agrado por las matemáticas por considerarla una asignatura importante para su vida y comprensión a las explicaciones de la maestra. Respecto a la atribución de éxitos a factores internos, estables y controlables como estudiar y entender las explicaciones de la maestra de acuerdo con la teoría de Weiner (1986), los estudiantes tienden a sostener el esfuerzo en el aprendizaje y estar motivados. Sin embargo, como se observó 
la valoración de los alumnos sobre su rendimiento sobrepasa la evaluación realizada por la maestra, un ejemplo de ello son los 13 alumnos que se encuentran en el nivel mínimo y dijeron ubicarse en el nivel satisfactorio, en este caso vale la pena reflexionar si esta interpretación obedece por un lado, a la sensación de sentirse capaces como respuesta a las regulaciones externas y por otro a la manera en la que en la clase se lleva a cabo el proceso de retroalimentación, recordemos que la maestra dadas las condiciones de enseñanza de que dispone, la realiza en forma grupal, señalando el error y explicando la solución correcta, lo cual no facilita a los estudiantes acercarse por sí mismos a la comprensión de sus aciertos y desaciertos mediante estrategias metacognitivas.

El bajo rendimiento es atribuido a la falta de comprensión a las explicaciones de la maestra, la indisciplina, intolerancia y desorden durante las clases y el incumplimiento de las tareas en casa por no saber cómo realizarlas. Para Weiner (1986) cuando los estudiantes atribuyen el fracaso o bajo rendimiento a aspectos externos y no controlables como en este caso el no comprender las explicaciones de la maestra y la falta de atención por la indisciplina en el aula, la motivación decrecerá y por lo tanto las expectativas de éxito académico, más aún si estos factores se perciben como estables (no van a cambiar), entonces los estudiantes tenderán a esperar los mismos resultados (Pozo, 2006). Otra consecuencia que vale la pena resaltar es que el atribuir constantemente el fracaso académico a causas externas, aunque protege la autoestima de los alumnos, genera la percepción de incapacidad de plantear y persistir en el logro de sus aspiraciones (Weiner, 1986). Esto tiene importantes consecuencias en la formación de la personalidad de los estudiantes, no solo en relación con la vida académica sino también en sus expectativas de asenso económico, social y bienestar como miembros de una comunidad.

Sin lugar a dudas, lo que revela la dinámica de las interacciones de los estudiantes y maestra de este grupo, y su relación con el aprendizaje es influido por las condiciones desfavorables para la enseñanza en este contexto social y escolar, los cuales favorecen la creencia de que el control externo de sus comportamientos puede ser entendido como algo normal. La ausencia de autonomía y reflexión sobre las causas de los comportamientos y sus consecuencias inciden negativamente en la construcción de conocimientos y habilidades necesarias para vivir en una sociedad en constante cambio y en donde la autonomía facilitaría la generación de expectativas de mejora y participación activa en la toma de decisiones en la vida personal y social.

\section{Conclusiones}

La presente investigación destaca la importancia que tiene la motivación intrínseca, extrínseca y social, así como las atribuciones causales del rendimiento académico en los procesos de enseñanza-aprendizaje, ya que no solo tienen incidencia en los resultados académicos, sino también en la formación de los estudiantes para afrontar la vida con autonomía y responsabilidad. Cuando el contexto social y escolar favorecen la motivación extrínseca en la mayoría de los alumnos como lo mostró este estudio, lo relevante va más allá de establecer relaciones motivación-rendimiento académico. Se trata de la posibilidad de reconocer las circunstancias en las que ocurre el desarrollo curricular se pueda romper el círculo de las condiciones sociales y escolares existentes que promueven la dependencia de los estudiantes al control externo, y crear nuevas para el aprendizaje activo y constructivo con el fin de promover el control del propio aprendizaje y mejorar las relaciones con el conocimiento matemático.

Los alcances de la motivación intrínseca en el rendimiento académico, además de proveer a los estudiantes satisfacción por el aprendizaje y favorecer los indicadores institucionales de aprovechamiento escolar, al estar estrechamente relacionada con la autonomía facilita el desarrollo de habilidades y la formación del estudiante para la construcción de conocimientos y por consiguiente un cierto empoderamiento en la comprensión y participación activa y reflexiva en el planteamiento de expectativas y la toma de decisiones en su propia vida y como ciudadano integrante de una comunidad.

Para el establecimiento de condiciones propicias para el aprendizaje activo, es indispensable que la comunidad educativa comprenda que cada alumno tiene que aprender por sí mismo, e incluir en el currículum oportunidades para aplicar el conocimiento por medio de la solución de problemas (Huber, 2008). Otro de los principios del aprendizaje activo es considerar que aprender es un proceso social, ya que también se aprende en interacción con otras personas (Huber, 2008). Alonso Tapia (2011) sugiere crear condiciones de trabajo en grupos cooperativos, entre sus beneficios es posible llegar a soluciones a pesar de los diferentes puntos de vista y aprender por observación del compañero, sus formas de trabajo, de enfrentarse a los problemas y recibir apoyo de los compañeros del grupo, todo lo cual tiene un efecto positivo sobre la motivación y sobre el aprendizaje. 
Dado que el ambiente que se vive en el interior del aula de clase incide positiva o negativamente en la motivación y en las atribuciones de éxito y fracaso en el rendimiento académico (Morales et al., 2016), es importante que este ambiente facilite el acceso a los conocimientos y habilidades para convivir constructivamente en el aula y en la sociedad, esto es posible si se enseña a los alumnos y docentes a interactuar y comunicarse para el diálogo igualitario, la solidaridad y la creación de sentido cultural (Flecha y Tortajada, 2013). Así también se sugiere que la evaluación docente forme parte de la enseñanza, de tal modo que permita a los estudiantes revisar las razones de sus errores o respuestas incorrectas para acercarlo al control de su propio aprendizaje, además que el docente puede estimular la autoestima y autonomía mediante comentarios que expresen los progresos conseguidos (Alonso Tapia, 2011; Deci y Ryan, 2015).

Estos hallazgos son de interés para la comunidad educativa y práctica docente, ya que el orientar las clases en ambientes de aprendizaje activo permite atender las dificultades que los estudiantes pueden presentar en los aspectos académicos y disciplinarios con el fin de reconocer sus necesidades e intereses, así como fomentar la autoestima y la autorregulación para ayudar a los estudiantes a desarrollar una relación constructiva consigo mismos y con el conocimiento matemático. Abellán Toledo y Herrada Valverde (2016) destacan que el docente con apoyo de la administración escolar es clave en la innovación educativa para beneficio del alumno. Para lograr lo anterior, los directivos y supervisores escolares tendrían que participar en conjunto con docentes, alumnos y padres de familia en la construcción de procesos de aprendizaje organizacional, en los cuales la investigación, reflexión y autocrítica pudieran generar conocimiento y proponer políticas y acciones para mejorar las condiciones para la enseñanza y el aprendizaje.

\section{Referencias}

Abellán Toledo, Y., y Herrada Valverde, R. (2016). Innovación educativa y metodologías activas en Educación Secundaria: La perspectiva de los docentes de lenguas castellana y literatura. Revista Fuentes, 18(1), 65-76. http://dx.doi.org/10.12795/revistafuentes.2016.18.1.04

Allen, M. (2013). Cuidado de sí mismo, equilibrio y perfil de aprendizaje de IB. Revista de Psicología Educativa, 1(2), 161-192. http://dx.doi.org/10.20511/pyr2013.v1n2.47

Alonso-Tapia, J. (mayo, 2011). Motivación para aprender, motivar para ser. Conferencia pronunciada en la VI Jornada Regional de ACLPP, Segovia, España. p 9-17 Recuperado de https://bit.ly/2yHUCMW.

Arana- Martínez, J., García-Meilán, J., Gordillo-León, F., y Carro-Ramos, J. (2010). Estrategias motivacionales y de aprendizaje para fomentar el consumo responsable de la escuela. Revista Electrónica de Motivación y Emoción, 13(3536), 19-39. Recuperado de https://bit.ly/2JTEWIX.

Bernal, C. (2010). Metodología de la investigación. Pearson Educación.

Caso-Niebla, J., y Hernández- Guzmán, L. (2007). Variables que inciden en el rendimiento académico de adolescentes mexicanos. Revista Latinoamericana de Psicología, 39(3), 487-501. Recuperado de https://bit.ly/2VdtrkO.

Deci, E., \& Ryan, R. (2015). Self-Determination Theory. International Encyclopedia of the Social \& Behavioral Sciences, 21, 486-491.

http://dx.doi.org/10.1016/B978-0-08-097086-8.26036-4

Del Valle-López, A. (2009). Formación del educador: Enfoque Competencial. Tendencias Pedagógicas, (14), 433 - 442. Recuperado de https://bit.ly/2VgNpv9.

Erazo-Santander, O. (2011). El rendimiento académico, un fenómeno de múltiples relaciones y complejidades. Revista Vanguardia Psicológica Clínica Teórica y Práctica, 2(2), 144-173. Recuperado de https://bit.ly/2JQdtb8.

Fernández-Domínguez, M. (2005). Más allá de la educación emocional. La formación para el crecimiento y el desarrollo personal del profesorado. Revista Interuniversitaria de Formación del profesorado, 19(3), 195-251. Recuperado de https://bit.ly/39ZoEJd.

Flecha, R., y Tortajada, I. (2013). Retos y salidas educativas en la entrada del siglo. En Imbernón, F. (Ed.), La educación en el siglo XXI. Los retos del futuro inmediato (p.13-27). Graó.

Flores, M., y González, O. (2014). El trabajo docente: Enfoques innovadores para el diseño de un curso. Trillas. Gimeno, J. (2013). En busca del sentido de la educación. Morata.

Goetz, J., y LeCompte, M. (1988). Etnografía y diseño cualitativo en investigación educativa. Ediciones Morata. González-Fernández, A. (2007). Modelos de motivación académica. Revista Electrónica de Motivación y Emoción, 10(25), s/p. Recuperado de https://bit.ly/2JW4Prs.

Huber, G. (2008). Aprendizaje activo y metodologías educativas. Revista de Educación, (número extraordinario) 59-81. Recuperado de https://bit.ly/2RHtmVN. Instituto Colombiano para el Fomento de la Educación Superior. (2017). Publicación de resultados Saber. Lamas, H. (2015). Sobre el rendimiento escolar. Propósitos y Representaciones, Revista de psicología educativa, 3(1), 313-386.

https://doi.org/10.20511/pyr2015.v3n1.74 
León, B. (2008). Atención plena y rendimiento académico en estudiantes de enseñanza secundaria. European Journal of Education and Psychology, 1(3), 17- 26.

https://doi.org/10.30552/ejep.v1i3.11

Maquilón-Sánchez, J., y Hernández-Pina, F. (2011). Influencia de la motivación en el rendimiento académico de los estudiantes de formación profesional. Revista Electrónica Interuniversitaria de Formación del Profesorado, 14(1), 81100. Recuperado de https://bit.ly/3c60Apl.

Merriam, S. (2015). Qualitative research. A guide to design and implementation. San Francisco, CA: Jossey Bass. Marchesi Ullastres, A. (2012). El bienestar de los docentes en tiempos de crisis. Revista Fuentes, 12, 9-12. Recuperado de https://bit.ly/2xoHcoV.

Miñano, P., y Castejón, J. (2008). Capacidad predictiva de las variables cognitivo-motivacionales sobre el rendimiento académico. Revista Electrónica de Motivación y Emoción, 11(28), s/p. Recuperado de https://bit.ly/2y0a86L.

Morales, L., Morales, V., y Holguín, S. (2016). Rendimiento académico. Revista Electrónica Humanidades, Tecnología y Ciencia del Instituto Politécnico Nacional, (15), 1-5. Recuperado de https://bit.ly/2K5WyRL.

Naranjo-Pereira, M.L. (2009). Motivación: perspectivas teóricas y algunas consideraciones de su importancia en el ámbito educativo. Revista Educación, 33(2), 153-170. Recuperado de https://bit.ly/2y8V5aD.

Pozo, J.I. (2006). Aprendices y maestros. La nueva cultura del aprendizaje. Madrid, España: Alianza Editorial. Salmerón-Pérez, H., y Gutierrez-Barojos, C. (2012). La competencia de aprender a aprender y el aprendizaje autorregulado. Revista de Currículum y Formación de Profesorado, 16(1), 5-13. Recuperado de https://bit.ly/3c5dO5D. Schunk, D. H. (2008). Metacognition, self-regulation, and self-regulated learning: Research recommendations. Educational Psychology Review, 20(4), 463-467. https://doi.org/10.1007/s10648-008-9086-3

Taylor, S.J., y Bogdan, R. (2006). Introducción a los métodos cualitativos de investigación. Paidós

Valenzuela, J., y Flores, M. (2012). Fundamentos de investigación educativa. Editorial Digital Tecnológico de Monterrey. Valenzuela, J., y Nieto, A. (2008). Motivación y pensamiento crítico: Aportes para el estudio de esta relación. Revista Electrónica de Motivación y Emoción, 11(28), s/p. Recuperado de https://bit.ly/34nw73B.

Valle, A., Rodríguez, S., Núñez, J., Cabanach, R., González-Pienda, J., y Rosario, P. (2010). Motivación y Aprendizaje Autorregulado. Interamerican Journal of Psychology, 44(1). 86-97. Recuperado de https://bit.ly/34p9IZ2.

Weiner, B. (1986). An Attributional Theory of Motivation and Emotion. Springer-Verlag. Zimmerman, B. (2010). Becoming a Self-Regulated Learner: An Overview. Theory Into Practice, 41(2), 64-70. https://doi.org/10.1207/s15430421tip4102_2 